\title{
Complex Bandpass Filtering for Coriolis Mass Flow Meter Signal Processing
}

\author{
Ming Li, Manus Henry \\ Department of Engineering Science \\ University of Oxford \\ Oxford, OX1 3PJ, UK. \\ manus.henry@eng.ox.ac.uk
}

\begin{abstract}
This paper explores the possible application of the complex bandpass filter in Coriolis mass flow meter signal processing. The method shows high robustness for frequency, amplitude and phase tracking under noisy conditions, such as those generated by two-phase (gas/liquid) flow. Simulations of the new technique are compared with existing academic and industrial signal processing solutions to evaluate their relative performance.
\end{abstract}

Keywords-Coriolis meter signal processing; complex bandpass filter; noisy condition;

\section{INTRODUCTION}

Coriolis Mass Flow Meter (CFM) is the most widely used metering technology which can directly measure the mass of the fluid flow [1]. A CFM consists of a mechanical, vibrating flowtube, and an electronic transmitter which generates measurement data and controls the flowtube vibration by generating an in-phase drive signal. Signal processing of CFM is thus challenging [2]: the amplitude, frequency, phase and phase difference of two sinusoidal sensor signals must be tracked to high precision, and with minimum delay, to ensure good flowtube control and accurate measurement. For example, Fig. 1 (from [3]) shows a timing diagram of the response of a transmitter to flow tube data. Here, the delays induced by $\mathrm{ADC}$ and DAC operations, filtering, data processing and phase synchronization combine to create a lag of three flowtube resonant cycles between the raw data and the

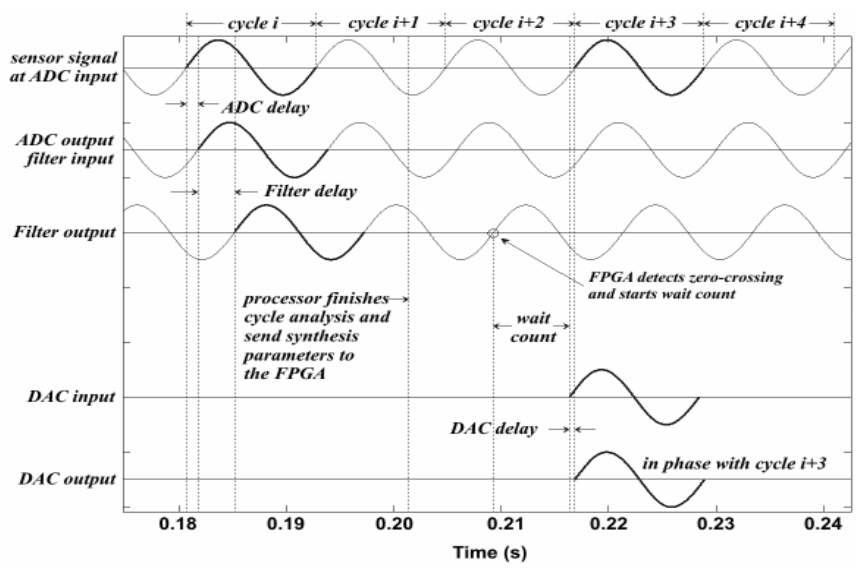

Fig. 1. Timing diagram of Coriolis signal processing (from [3]). drive signal response. Under normal operating conditions, such a delay rarely causes any difficulty. However, increasingly Coriolis meters are being applied to more difficult fluids, in particular liquid/gas mixtures [1]. In these conditions the frequency and amplitude of the sensor signals vary much more rapidly, and the ability of tracking algorithms to follow these changes with high accuracy and minimum delay becomes increasingly important. While the system described in [3] can indeed maintain flowtube vibration and generate useful measurement data in the presence of two-phase flow, there is significant scope for improving performance by finding fast sinusoid tracking algorithms which are robust to noise and rapid parameter variation.

A range of CMF signal processing techniques have been developed in academia and industry ([4]-[7]). Techniques include Adaptive Notch Filters (ANF), Hilbert Transforms, the Discrete-Time Fourier Transform (DTFT), and Digital Phase Locked Loops (DPLL). However, previous work has mostly excluded an analysis of dynamic response and performance under noisy conditions. In this paper a new approach, employing the Complex Bandpass Filter (CBP), is used to track frequency, amplitude and phase simultaneously. The CBP performance is compared with ANF and Hilbert transform methods in simulations of noisy and rapidly-varying sensor signal data.

\section{COMPLEX BANDPASS FILTER}

The Complex Bandpass Filter is used in a wide range of communication systems ([8], [9]) and also in the biomedical area [10]. Conventionally, a digital CBP can be simply derived from a low-pass filter by multiplying a 'shift' complex factor $e^{j \theta_{c}}$ to the real coefficients of the low-pass filter. This is equivalent to applying the substitution [11]:

$$
z^{-1} \rightarrow z^{-1} e^{j \theta}=z^{-1}(\cos \theta+j \sin \theta)
$$

so that the transfer function becomes:

$$
\begin{aligned}
& H_{\text {real }}(z) \stackrel{z^{-1}=z^{-1}(\cos \theta+j \sin \theta)}{\longrightarrow} H_{\text {complex }}(x) \\
& =H_{R}(z)+j H_{I}(z)
\end{aligned}
$$

The bandwidth of the CBF is twice that of the original filter, but other properties of the filter remain the same. The CBP's 
central frequency is shifted to the desired value of $\theta$. Fig. 2 shows a pole-zero plot illustrating the change of poles when a CBF is created from a first-order real low-pass filter [11]:

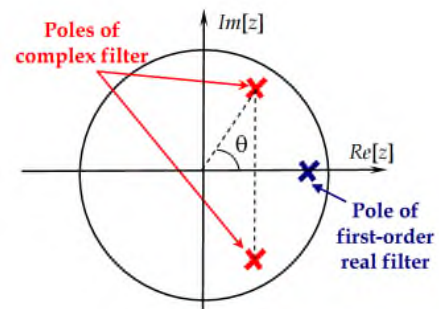

Fig. 2. Pole rotation of a first-order real transfer function to complex filter (from [11])

Passing a (real) input signal through the newly formed CBP generates Hilbert pair (i.e. orthogonal) outputs. The Hilbert pair form an analytic signal so that amplitude, frequency and phase can be readily calculated. Our design begins with an elliptic low-pass filter since it can achieve a fast cutoff using a lower order than other conventional filters. The range of resonant frequencies for the intended CMF flowtube is $80-100 \mathrm{~Hz}$. Accordingly, the original low pass filter was designed as a $5^{\text {th }}$ order elliptic filter with a $2 \mathrm{kHz}$ sampling frequency, $0.1 \mathrm{~dB}$ maximum passband ripple, and a $50 \mathrm{~Hz}$ passband edge frequency. Having created this filter, the coefficients are multiplied by the complex $90 \mathrm{~Hz}$ 'shift' factor, resulting in a new, complex bandpass filter, as shown in Fig. 3:

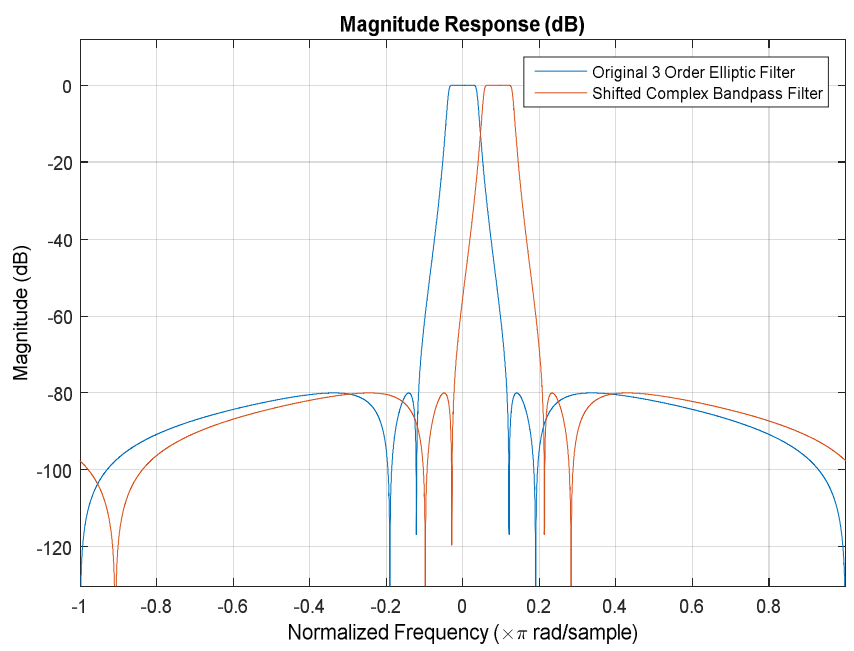

Fig. 3. Original low-pass filter and CBP filter frequency response.

Having created the CBP, the amplitude, frequency, phase and phase difference of the two Coriolis sensor signals are calculated as follows. Note that as parameters values are calculated from the CBP generated analytic form, the equations are similar to those used with the Hilbert transform approach [5]. We assume the input sensor signals are:

$$
\begin{aligned}
& x_{1}=A_{1} \sin (\omega t+\phi / 2) \\
& x_{2}=A_{2} \sin (\omega t-\phi / 2)
\end{aligned}
$$

where $A_{1}$ and $A_{2}$ are the amplitudes of the two sensor signals, $\omega$ is their common frequency, and $\phi$ is the phase difference between them. Passing the signals through CBP we obtain complex analytic signals as follows:

$$
\begin{aligned}
& x_{1 a}=A_{1}[\cos (\omega t+\phi / 2)+i \sin (\omega t+\phi / 2)]=A_{1} e^{j(\omega t+\phi / 2)} \\
& x_{2 a}=A_{2}[\cos (\omega t-\phi / 2)+i \sin (\omega t-\phi / 2)]=A_{2} e^{j(\omega t-\phi / 2)}
\end{aligned}
$$

Then taking the conjugate terms we get:

$$
x_{1 a} \times \overline{x_{2 a}}=A_{1} e^{j(\omega t+\phi / 2)} \times A_{2} e^{-j(\omega t-\phi / 2)}=A_{1} \times A_{2} e^{j \phi}
$$

Assuming here for brevity that the two input signals amplitude are the same $\left(A_{1}=A_{2}\right)$, equation (5) is simplified to:

$$
x_{1 a} \times \overline{x_{2 a}}=A^{2}(\cos \phi+j \sin \phi)
$$

The phase difference is then calculated using:

$$
\phi=\arg \left(x_{1 a} \times \overline{x_{2 a}}\right)
$$

Also the amplitude can be obtained using:

$$
A_{1}=A_{2}=a b s\left(x_{1 a}\right)=a b s\left(x_{2 a}\right)
$$

The frequency is derived from the change of phase between adjacent samples from the first sensor signal:

$$
\begin{aligned}
\overline{x_{1 a}(\mathrm{n}-1)} \times x_{1 a}(\mathrm{n}) & =a e^{-j\left(\omega t_{n-1}+\phi / 2\right)} \times a e^{j\left(\omega t_{n}+\phi / 2\right)} \\
& =a^{2} e^{j\left(\omega t_{n}-\omega t_{n-1}\right)}
\end{aligned}
$$

followed by:

$$
\omega t_{n}-\omega t_{n-1}=\arg \left(\overline{x_{1 a}(\mathrm{n}-1)} \times x_{1 a}(\mathrm{n})\right)
$$

Converting into $\mathrm{Hz}$ :

$$
f=\frac{\left(\omega t_{n}-\omega t_{n-1}\right) \times F s}{2 \pi}
$$

where $F_{S}$ is the sampling frequency.

In order to evaluate the performance of the algorithm under difficult conditions, criteria have been selected for simulating realistic parameter changes. Two difficult conditions are considered in this paper: empty-to-full, and continuous twophase flow (which is discussed later in the paper).

The disturbance caused when a flowtube, initially empty, is rapidly filled with liquid, for example in a batching application, has been a significant Coriolis problem. This transition results in rapid changes in all parameters simultaneously. Here in simulation the frequency is changed from $100 \mathrm{~Hz}$ down to $85 \mathrm{~Hz}$ (reflecting an increase in density from air to liquid), the amplitude is changed from $0.3 \mathrm{~V}$ down to $0.05 \mathrm{~V}$ (reflecting an increase in flowtube damping) and the phase difference between the two sensors is changed from $0^{\circ}$ up to $4^{\circ}$ (reflecting a change from no flow up to a high flow rate). All of these changes occur simultaneously over a period of only $0.5 \mathrm{~s}$, reflecting a typical 'fast fill' of a real flowtube. To assist analysis, these parameter values changes are applied linearly throughout the $0.5 \mathrm{~s}$, although in reality the changes would be non-linear and accompanied by significant additional noise.

In our simulation, the sensors signals are passed through the CBP and the calculations given above are used to track amplitude, frequency and phase difference. Fig. 4, Fig. 5 and Fig. 6 show the performance of each parameter: 

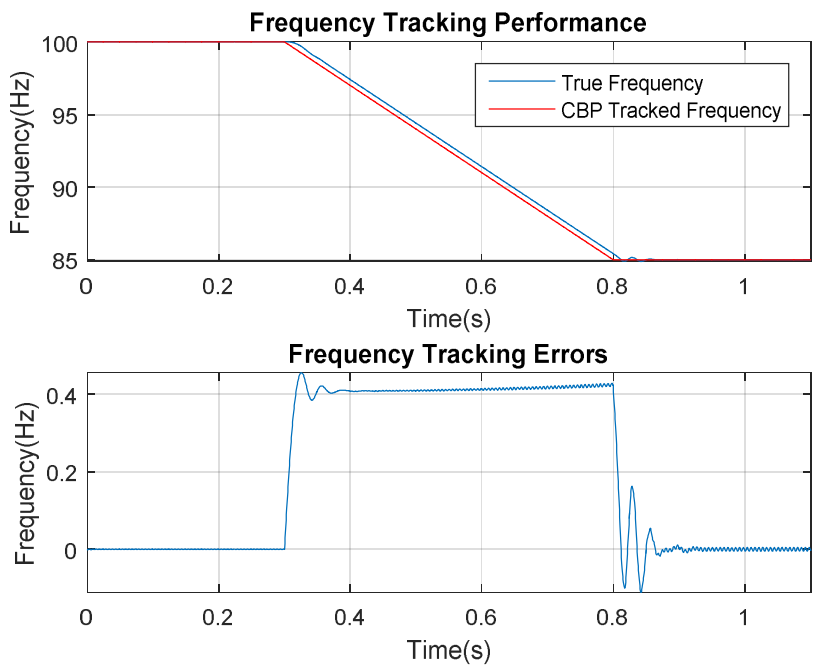

Fig. 4. Frequency tracking performance of CBP
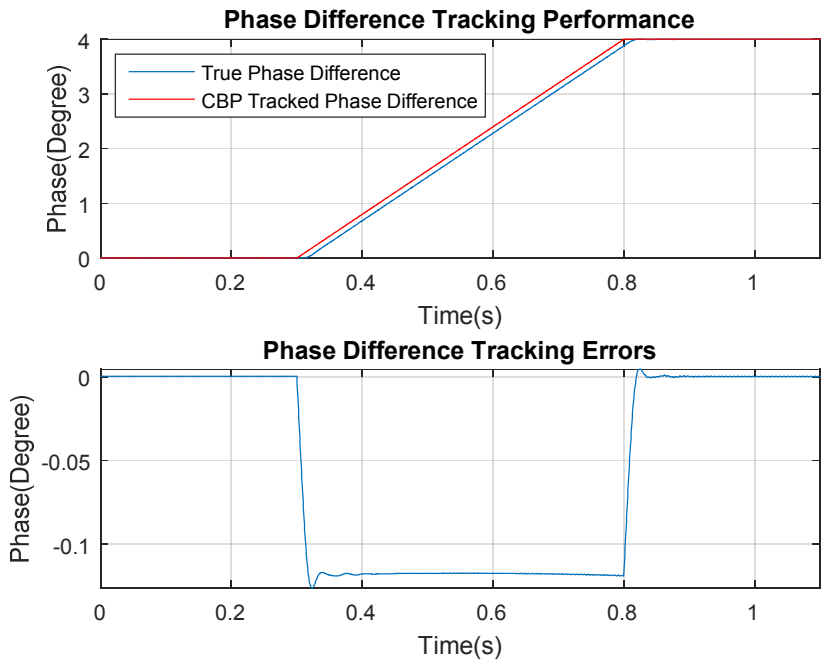

Fig. 5. Phase difference tracking performance of CBP
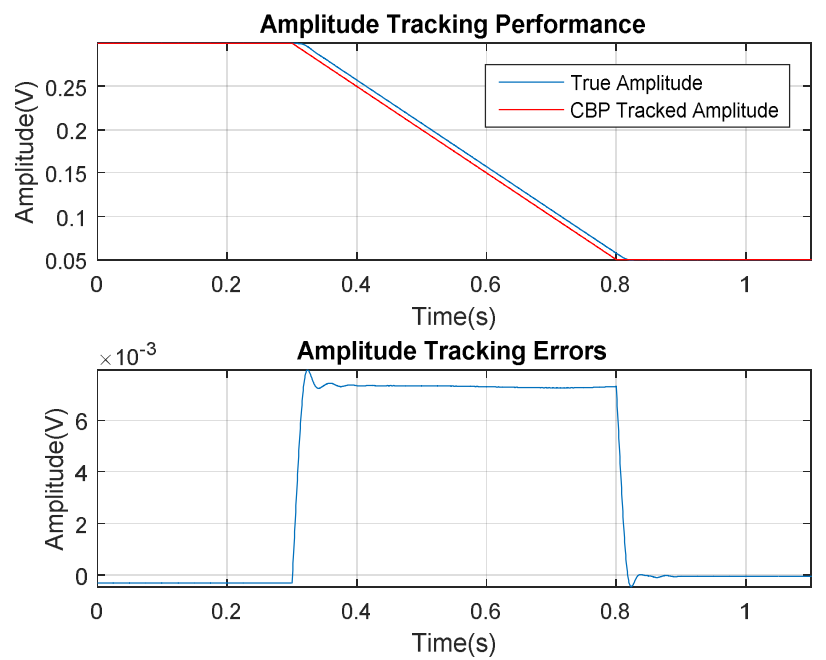

As is clear from the figures, the CBP generates little noise when subject to rapidly varying input signals. The main error feature is the time delay between the true and tracked values, which is roughly $15 \mathrm{~ms}$ for each parameter, and is caused by the group delay of the filter.

\section{SIMULATION OF TWO-PHASE FLOW CONDITIONS}

Of the techniques discussed in section I, ANF combined with DTFT, and the Hilbert transform method have been found to perform best [4]. Accordingly, here we compare CBP with these two methods under simulated two-phase flow conditions.

\section{A. Modified Random Walk Model for two-phase flow}

In [18], the authors used a Random Walk Model (RWM) to generate time-varying input sensor signal as follows:

$$
\begin{aligned}
& y_{1}(n)=A(n) \sin \left[2 \pi n f(n) / f_{s}+\phi(n) / 2\right]+\sigma_{e 1} \cdot e_{1}(n) \\
& y_{2}(n)=A(n) \sin \left[2 \pi n f(n) / f_{s}-\phi(n) / 2\right]+\sigma_{e 2} \cdot e_{2}(n) \\
& A(n)=A(n-1)+\sigma_{A} \cdot e_{A}(n) \\
& f(n)=f(n-1)+\sigma_{f} \cdot e_{f}(n) \\
& \phi(n)=\phi(n-1)+\sigma_{\phi} \cdot e_{\phi}(n)
\end{aligned}
$$

where $y_{1}$ and $y_{2}$ are the simulated sensor signals, with common but time varying amplitude $A$, frequency $f$, and phase difference $\phi$, and where $f_{s}$ is the sample rate for simulation. $e_{1}(n), e_{2}(n), e_{A}(n), e_{f}(n)$ and $e_{\phi}(n)$ are uncorrelated white noise processes. $\sigma_{e 1}, \sigma_{e 2}$ are the gains for the noise on each input signal, and $\sigma_{A}, \sigma_{f}$ and $\sigma_{\phi} \sigma_{\phi}$ are the noise gains for each sine wave parameter. Together, these parameters determine the evolution of the simulated signal over time.

However, this model has the disadvantage that there are no limits on the instantaneous rate of change of the parameter values, as the random sequences are unfiltered. In any real CMF there are physical limitations on the rate of change of parameter values due to mechanical inertia, limited fluid velocities and so on. To provide a more realistic simulation, we use a Modified Random Work Model (MRWM) by defining boundaries as follows:

$$
\begin{aligned}
& y_{1}(n)=A_{l}(n) \sin \left[2 \pi n f_{l}(n) / f_{s}+\phi_{l}(n) / 2\right]+\sigma_{e 1} \cdot e_{1}(n) \\
& y_{2}(n)=A_{l}(n) \sin \left[2 \pi n f_{l}(n) / f_{s}-\phi_{l}(n) / 2\right]+\sigma_{e 2} \cdot e_{2}(n) \\
& A_{l}(n)=H_{A}(n)^{*} A(n) \\
& f_{l}(n)=H_{f}(n)^{*} f(n) \\
& \phi_{l}(n)=H_{\phi}(n)^{*} \phi(n) \\
& A(n)=\frac{\left(A_{\max }+A_{\min }\right)}{2}+\frac{\left(A_{\max }-A_{\min }\right)}{2} \cdot e_{A}(n) \\
& f(n)=\frac{\left(f_{\max }+f_{\min }\right)}{2}+\frac{\left(f_{\max }-f_{\min }\right)}{2} \cdot e_{f}(n) \\
& \phi(n)=\frac{\left(\phi_{\max }+\phi_{\min }\right)}{2}+\frac{\left(\phi_{\max }-\phi_{\min }\right)}{2} \cdot e_{\phi}(n)
\end{aligned}
$$

Fig. 6. Amplitude tracking performance of $\mathrm{CBP}$ 
where $e_{1}(n), e_{2}(n), e_{A}(n), e_{f}(n)$ and $e_{\phi}(n)$ remain uncorrelated white noise sequences, $\sigma_{e 1}$ and $\sigma_{e 2}$ are gain factors for the input noise, while $H_{A}(n), H_{f}(n)$ and $H_{\phi}(n)$ are lowpass filters to limit the rate of change of parameter values. $A_{\max }, A_{\min }$ are maximum and minimum limits of amplitude, where $f_{\max }, f_{\min }, \phi_{\min }$ and $\phi_{\max }$ are the same for frequency and phase difference.

The boundary limited amplitude, frequency and phase difference are generated via white noise process and then passed through a low-pass filter to constraint the rate of change. Then based on the filtered parameters, the two sensor signals are generated. Fig. 7 compares the output of MRWM with that of RWM using the following constraints: $f_{\min }=85$ $\mathrm{Hz}, f_{\text {max }}=100 \mathrm{~Hz}, A_{\text {min }}=0.05 \mathrm{~V}, A_{\text {max }}=0.35 \mathrm{~V}, \phi_{\text {min }}=0^{\circ}$ and $\phi_{\max }=4^{\circ}$.
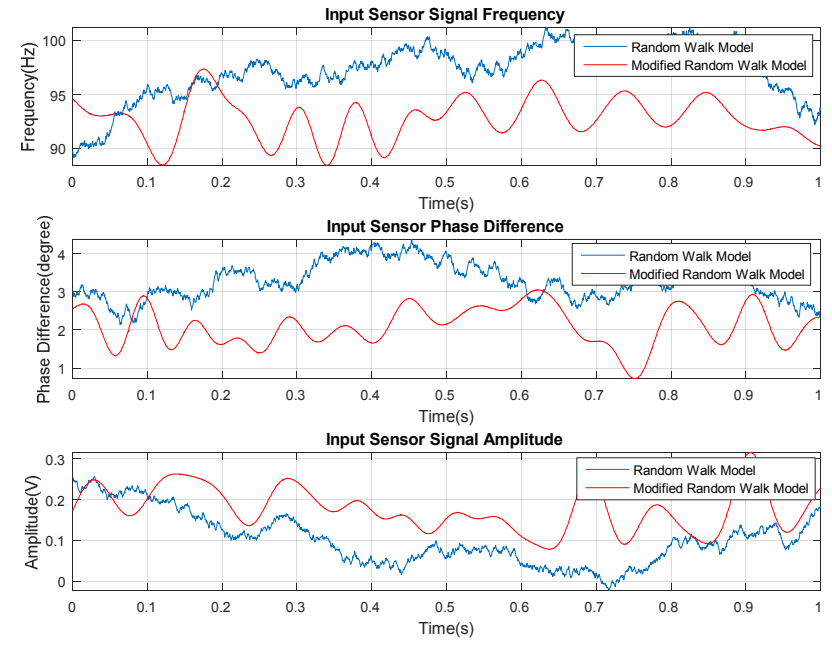

Fig. 7. Comparison between RWM and MRWM

From the figure, both the RWM and MRWM range over the desired parameter values in a random fashion. However MRWM excludes high frequency variations in parameter values which are physically unrealistic, but which will influence the error statistics and hence the performance evaluation of each of the measurement techniques.

\section{B. Comparing CBP with Hilbert transform and $A N F$}

Based on signals generated using MRWM, simulations of CBP have been carried out alongside the ANF and Hilbert transform techniques. Fig. 8 shows the corresponding sensor data supplied to the tracking algorithms.

Results are presented for the following methods:

- ANF, Hilbert, CBP for frequency tracking;

- DTFT (ANF based frequency), Hilbert, CBP for phase difference tracking; and

- DTFT (ANF based frequency), Hilbert, CBP for amplitude tracking.
The DTFT (ANF based frequency) and Hilbert implementations are the same as those described in an earlier paper [4]. Figs. $9-14$ show the tracking performance of amplitude, frequency and phase difference for each of the methods, for both noise free and for $20 \mathrm{~dB}$ Signal-to-Noise Ratio (SNR) simulations. In each case the tracked values are shown in the upper graph, with the residual errors below.

The frequency tracking error has high bandwidth for the Hilbert method, especially when input noise is added (Fig. 13); the error has low bandwidth for the ANF method, while CBP can accurately track frequency even with high noise.

For phase difference, the Hilbert method shows good tracking with some time delay in the noise free case, but it suffers from increasing error in noisy conditions. The DTFT results are dominated by the frequency errors of the ANF upon which it relies. CBP performs well for phase difference tracking both in noise free and noisy condition.

Similarly, for amplitude, the DTFT method shows large errors while the Hilbert method tracks relatively well with some time delay in noise free condition and shows reduced performance in noisy conditions. CBP again performs well both in noise free and noisy conditions.

Normally the Hilbert method is used in conjunction with a sharp bandpass filter to improve the noise performance. But this introduces extra delay which worsens the dynamic performance. So here only the pure Hilbert method is adopted in order to compare performance.
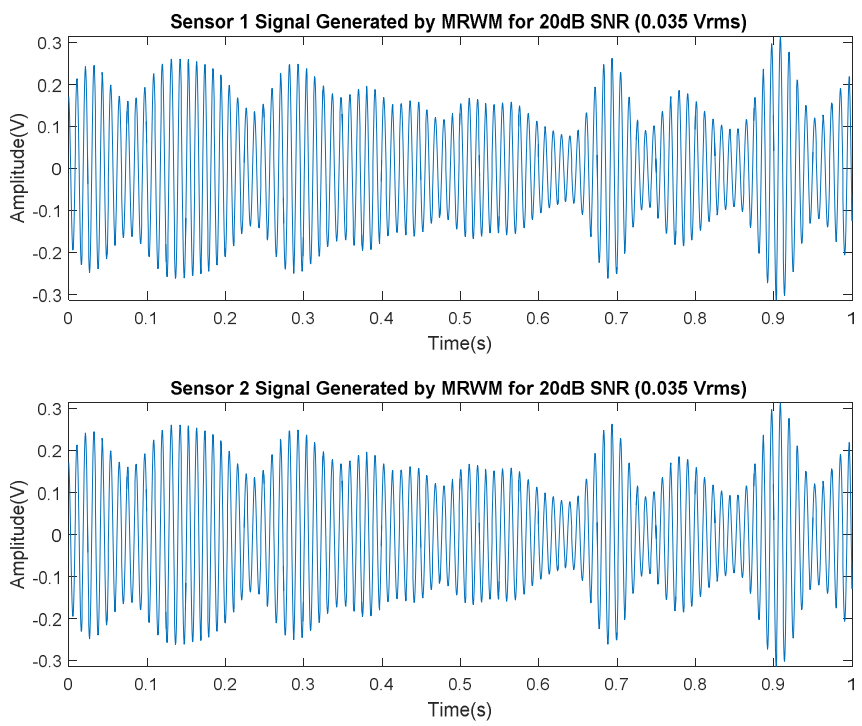

Fig. 8. Sensor 1 and sensor 2 signal generated by MRWM 

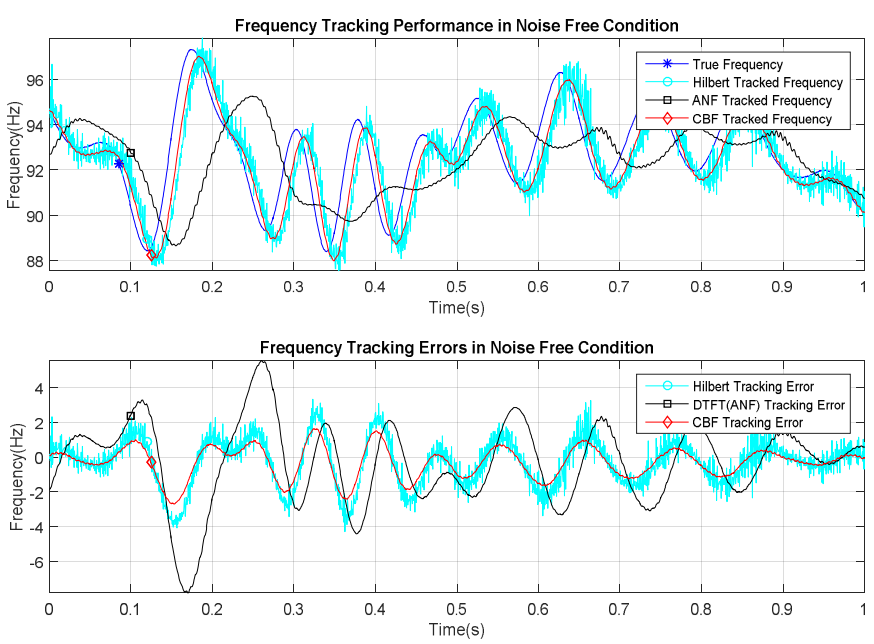

Fig. 9. Frequency tracking performance - no noise
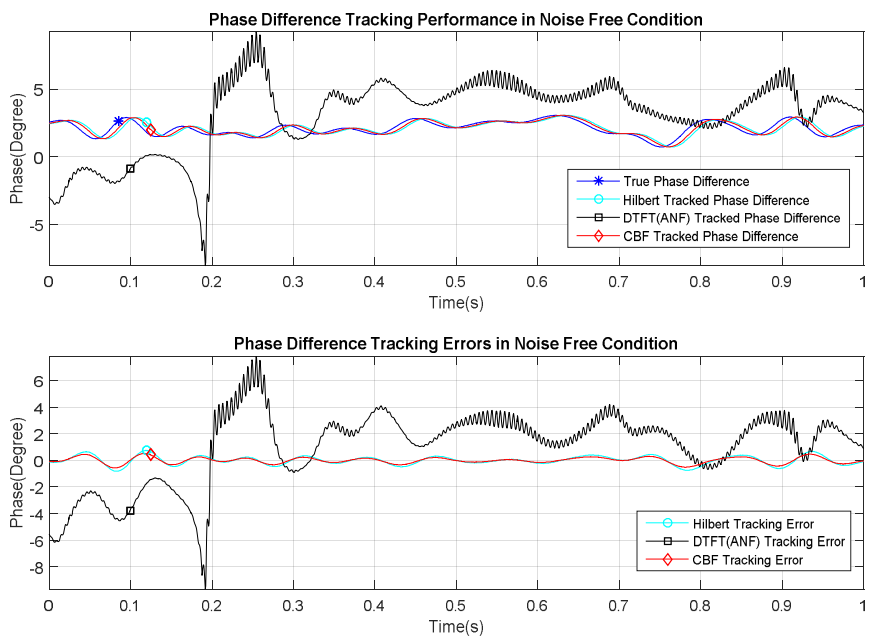

Fig. 10. Phase difference tracking performance - no noise
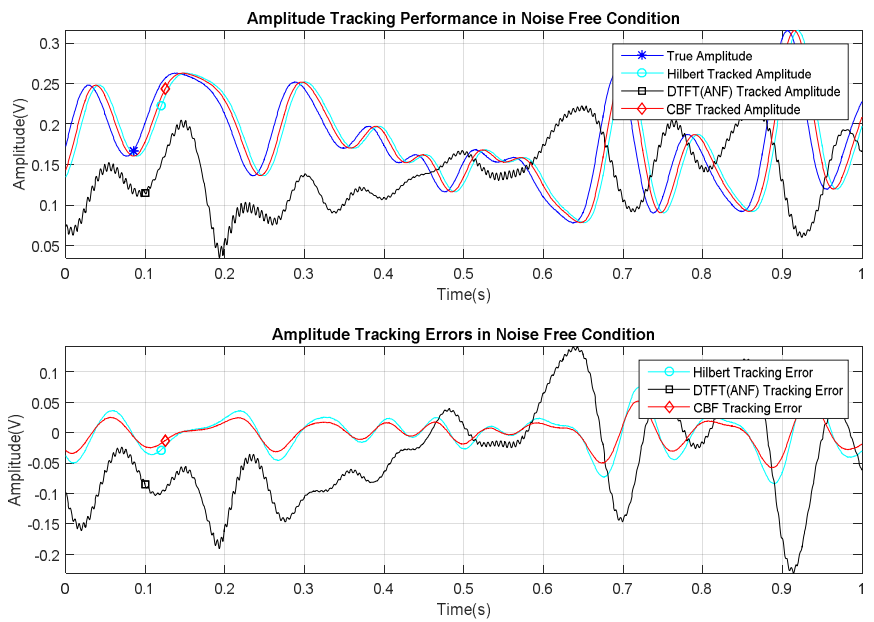

Fig. 11. Amplitude tracking performance - no noise
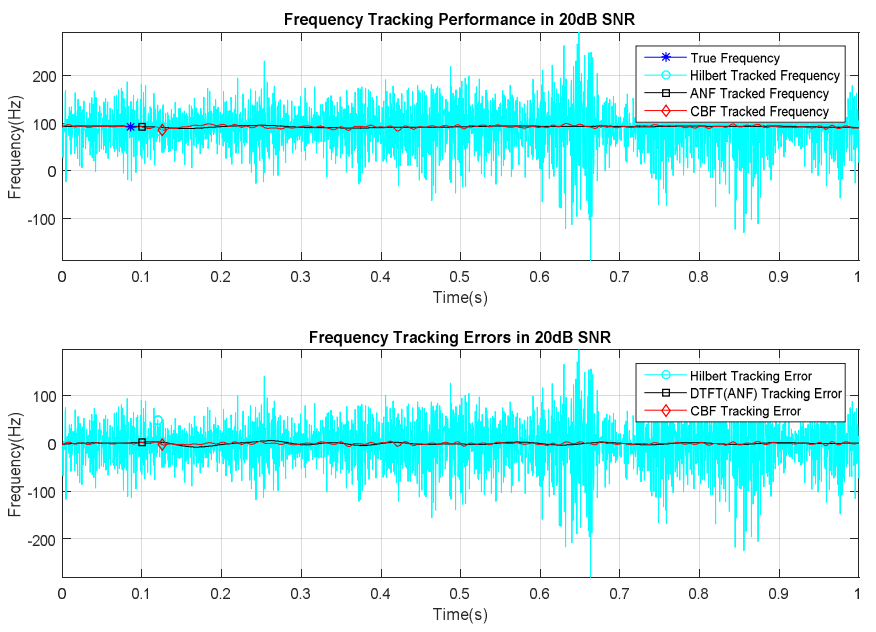

Fig. 12. Frequency tracking performance with $20 \mathrm{~dB}$ SNR $(0.035 \mathrm{Vrms})$
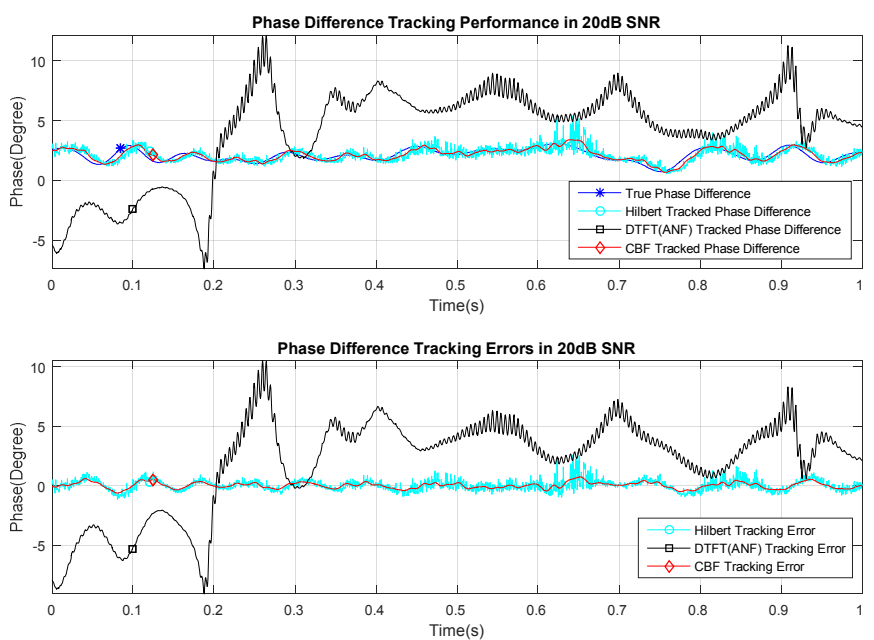

Fig. 13. Phase difference tracking performance with $20 \mathrm{~dB}$ SNR $(0.035 \mathrm{Vrms})$
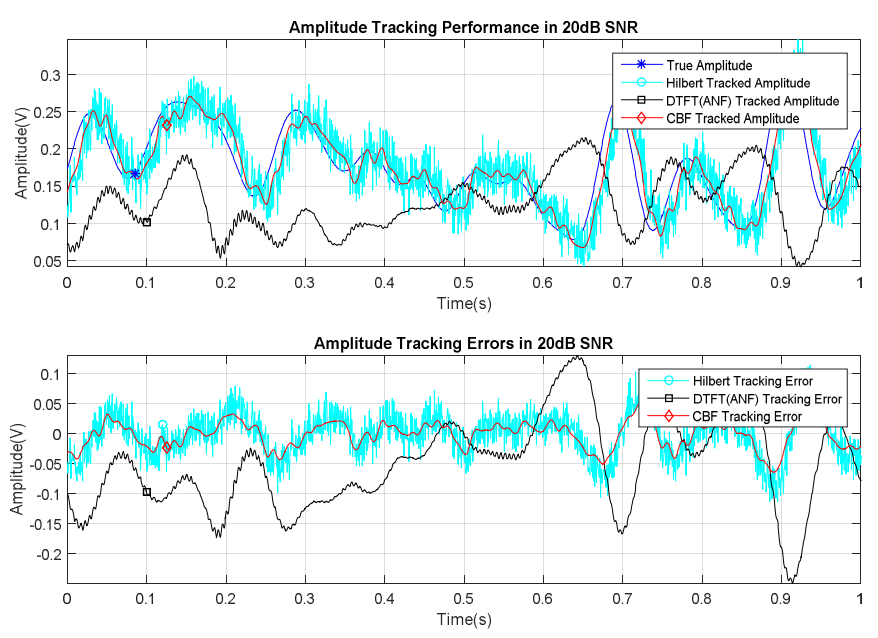

Fig. 14. Amplitude tracking performance with 20dB SNR (0.035 Vrms) 


\section{Quantified Error Performance}

To evaluate each method's performance numerically, Table I shows the Root Mean Squared Error (RMSE), calculated by:

$$
R M S E=\sqrt{\frac{1}{n} \sum_{i=1}^{n}(\hat{Y}(n)-Y(n))^{2}}
$$

where $Y(n)$ and $Y(n)$ are the true and estimated values. The results are shown in Table 1 for the noise free and 20dB SNR experiments.

TABLE I. QuANTIFIEd Tracking Performance

\begin{tabular}{|l|r|r|c|}
\hline \multicolumn{4}{|c|}{ RMSE in Noise Free Experiment } \\
\hline $\begin{array}{l}\text { Method and } \\
\text { Parameter }\end{array}$ & DTFT (ANF) & Hilbert & CBP \\
\hline $\begin{array}{l}\text { Frequency RMSE } \\
\text { (Hz) }\end{array}$ & 2.3650 & 1.3799 & 0.9292 \\
\hline $\begin{array}{l}\text { Phase Difference } \\
\text { RMSE ( })\end{array}$ & 2.9230 & 0.3254 & 0.2247 \\
\hline $\begin{array}{l}\text { Amplitude RMSE } \\
\text { (V) }\end{array}$ & 0.08847 & 0.03161 & 0.02167 \\
\hline \multicolumn{1}{|c|}{ RMSE in 20dB SNR Experiment } \\
\hline $\begin{array}{l}\text { Method and } \\
\text { Parameter }\end{array}$ & DTFT (ANF) & Hilbert & CBP \\
\hline $\begin{array}{l}\text { Frequency RMSE } \\
\text { (Hz) }\end{array}$ & 2.3664 & 56.6221 & 1.7516 \\
\hline $\begin{array}{l}\text { Phase Difference } \\
\text { RMSE ( }\end{array}$ & 3.5563 & 0.4548 & 0.2502 \\
\hline $\begin{array}{l}\text { Amplitude RMSE } \\
\text { (V) }\end{array}$ & 0.08848 & 0.03745 & 0.02280 \\
\hline
\end{tabular}

\section{CONCLUSION}

This paper has described the Complex Bandpass Filter algorithm and has applied it to CMF signal processing. The CBP can be derived from a simple low-pass filter with a selectable central frequency and bandwidth. The calculation is simple and computational cost is small. Due to the nature of bandpass filter, the CBP can not only track amplitude, frequency and phase difference at the same time, but it also applies strong noise suppression, which is increasingly important in CMF applications. Finally, its tracking performance is generally superior to that of the DTFT (ANF based) and Hilbert transform techniques.

For future work, further performance improvements (particularly reduced time delay) will be sought by using a Monte-Carlo search algorithm to design the low-pass filter for minimum group delay. The authors' research group is currently developing a next-generation Coriolis transmitter architecture, which will provide a real-time test bed for the new algorithms.

\section{REFERENCES}

[1] T. Wang and R. Baker, "Coriolis flowmeters: a review of developments over the past 20 years, and an assessment of the state of the art and likely future directions," Flow Meas. Instrum., vol. 40, pp. 99-123, Dec. 2014.

[2] J. Ruoff, W. Gauchel, and H. Kück, "Advances in Signal Acquisition and Signal Processing of Coriolis Flow Meters," Procedia Eng., vol. 87, pp. 1585-1588, 2014.

[3] M. Zamora and M. P. Henry, "An FPGA Implementation of a Digital Coriolis Mass Flow Metering Drive System," IEEE Trans. Ind. Electron., vol. 55, no. 7, pp. 2820-2831, Jul. 2008.

[4] M. Li and M. P. Henry, "Signal Processing Methods for Coriolis Mass Flow Metering in Two-Phase Flow Conditions," in 2016 IEEE International Conference on Industrial Technology (ICIT), 2016.

[5] G. R. Duffill, S. M. Jones, and A. T. Patten, "Meter electronics and methods for determining a liquid flow fraction in a gas flow material," U.S. Patent 7974792, 05-Jul-2011.

[6] N. Penghhui and T. Yaqing, "A Method for Signal Processing of Coriolis Mass Flowmeter Based on Combining AR Model Spectrum Estimation with DFT," in 2006 IEEE Instrumentation and Measurement Technology Conference Proceedings, 2006, pp. 1417-1420.

[7] X. Wenfu and X. Kejun, "A Signal Processing Method Based on AFF and SGA for Coriolis Mass Flowmeters," Acta Metrol. Sin., vol. 28, no. 1, pp. 48-51, 2007.

[8] K. W. Martin, "Complex Signal Processing is Not Complex," IEEE Trans. Circuits Syst. I Regul. Pap., vol. 51, no. 9, pp. 1823-1836, Sep. 2004.

[9] K. Wang, "The implementation of MSK based on DSP and complex analytic bandpass filter," in 2011 IEEE International Conference on Computer Science and Automation Engineering, 2011, vol. 1, pp. 229232.

[10] A. Link, M. Burghoff, A. Salajegheh, D. Poeppel, L. Trahms, and C. Elster, "Comparing a template approach and complex bandpass filtering for single-trial analysis of auditory evoked M100.," Biomed. Tech. (Berl)., vol. 52, no. 1, pp. 106-10, Feb. 2007.

[11] Z. Nikolova, G. Stoyanov, G. Iliev, and V. Poulkov, Digital Filters. InTech, 2011.

[12] R. Cheesewright, C. Clark, A. Belhadj, and Y. Y. Hou, "The dynamic response of Coriolis mass flow meters," J. Fluids Struct., vol. 18, no. 2, pp. 165-178, Sep. 2003.

[13] M. Henry, Z. ME, C. C, R. Cheesewright, and W. Mattar, "The dynamic response of Coriolis mass flow meters: Theory and applications," in 4th Annual Emerging Technologies Conference, ISA EXPO 2004, 2004.

[14] C. Clark, R. Cheesewright, and S. Wang, "Prediction of the Dynamic Performance of Fast Response Coriolis Meter Systems," IEEE Trans. Instrum. Meas., vol. 57, no. 1, pp. 95-99, Jan. 2008.

[15] C. Clark, R. Cheesewright, S. Wang, M. Henry, M. Zamora, and M. Tombs, "A radically new dynamic response capability for Coriolis flow meters," Sensors Actuators A Phys., vol. 123-124, pp. 54-62, Sep. 2005.

[16] C. Clark and R. Cheesewright, "Experimental determination of the dynamic response of Coriolis mass flow meters," Flow Meas. Instrum., vol. 17, no. 1, pp. 39-47, Mar. 2006.

[17] M. P. Henry, C. Clark, M. Duta, R. Cheesewright, and M. Tombs, "Response of a Coriolis mass flow meter to step changes in flow rate," Flow Meas. Instrum., vol. 14, no. 3, pp. 109-118, Jun. 2003.

[18] Y. Tu, H. Yang, H. Zhang, and X. Liu, "CMF Signal Processing Method Based on Feedback Corrected ANF and Hilbert Transformation,” Meas. Sci. Rev., vol. 14, no. 1, 2014. 